\title{
Hybrid coronary revascularization for the treatment of multivessel coronary artery disease
}

\author{
Michael O. Kayatta ${ }^{1}$, Michael E. Halkos ${ }^{1}$, John D. Puskas ${ }^{2}$ \\ ${ }^{1}$ Division of Cardiothoracic Surgery, Department of Surgery, Emory University, Atlanta, GA, USA; ${ }^{2}$ Department of Cardiovascular Surgery, Mount \\ Sinai Saint Luke's, New York, NY, USA \\ Correspondence to: John D. Puskas, MD. Professor and Chairman, Department of Cardiovascular Surgery, Mount Sinai Saint Luke's, 1111 Amsterdam \\ Ave, 6th Floor Babcock Building, New York, NY, USA. Email: john.puskas@mountsinai.org.
}

\begin{abstract}
Coronary artery disease (CAD) has typically been treated either medically, with percutaneous coronary intervention (PCI), or with coronary artery bypass grafting (CABG). As advances in stent technology and minimally invasive surgery have developed, a third option has emerged: hybrid coronary revascularization (HCR). In HCR, minimally invasive CABG and PCI are both employed to treat a single patient, often during the same hospital stay. Patients appropriate for this technique vary widely, from low-risk patients with low SYNTAX lesions outside the left anterior descending artery (LAD), to high-risk patients with multiple comorbidities who are felt by the heart team to benefit most by avoiding a sternotomy. Across both our experience and other series in the literature, mortality with HCR is around 1\%. Hospital length of stay is less than one week, and typically less than after conventional CABG, but longer than with isolated PCI. Return to baseline activity is substantially shorter after minimally invasive CABG compared to conventional CABG due to the avoidance of a sternotomy; deep sternal wound infections are entirely avoided. Mid-term need for repeat revascularization may be higher with HCR, though randomized data are lacking. In conclusion, HCR is an evolving method to treat multivessel CAD with favorable early results in high volume centers, though growth in the field is limited by surgical experience and success with minimally invasive techniques.
\end{abstract}

Keywords: Hybrid; robotics; coronary artery bypass grafting (CABG); percutaneous coronary intervention (PCI); minimally invasive direct coronary artery bypass (MIDCAB)

Submitted Mar 15, 2018. Accepted for publication May 29, 2018.

doi: $10.21037 /$ acs.2018.06.09

View this article at: http://dx.doi.org/10.21037/acs.2018.06.09

\section{Introduction}

Patients with coronary artery disease (CAD) affecting both the left anterior descending artery (LAD) and at least one other coronary artery are potential candidates for hybrid coronary revascularization (HCR). Patients with complex LAD lesions that may not be ideal for stenting, along with lesions in other coronary arteries that are easily stented, represent the ideal candidates for HCR, as they can benefit from the longevity of the left internal mammary artery anastomosed to the LAD (LIMA-LAD). These patients avoid a sternotomy, avoid the use of saphenous vein grafts (SVG) and recover more quickly from surgery. Minimally invasive coronary artery bypass grafting (MICS-CABG) was developed more than two decades ago using direct visualization methods, such as a thoracotomy or partial sternotomy, to harvest the LIMA from the chest wall and create the LIMA-LAD anastomosis. Several of these techniques are still widely used today, though the addition of advanced visualization and robotics has increased surgical options. During this time frame, there has been a steady advance in percutaneous coronary intervention (PCI) technologies. These include changes in delivery (e.g., radial artery), to better sheaths, wires, and catheters, improved imaging and functional assessments (e.g., fractional flow reserve), as well as drastic improvements in stent technology. These PCI advances have greatly improved the safety and durability of these procedures. 


Table 1 Patient selection for HCR
Indications
Significant LAD lesion
PCI appropriate for lesions other than LAD
Acute infarction with non-LAD culprit
Patient not a good candidate for multivessel CABG
Poor right coronary or circumflex arteries for bypass
Porcelain aorta
Lack of acceptable conduits (e.g., vein stripping)
Patient desire for minimally invasive procedure
Marginal candidates
Difficult LAD for bypass (small, calcified, intramyocardial)
Moderate SYNTAX non-LAD disease
Previous chest surgery
Significant obesity
Moderate to severe COPD (single-lung ventilation)
Absolute contraindications
Emergency operations
descending artery; CABG, coronary artery bypass grafting.
COPD, chronic obstructive pulmonary disease.
Paity

While multiple clinical trials have demonstrated superior freedom from angina and revascularization with CABG over isolated PCI (1-3), it is not clear whether this benefit comes from the LIMA-LAD alone, which has a 10-15-year patency of greater than $95 \%$ (4), or whether SVG also contribute to this, since they have a patency of as low as $60 \%$ at 1 year (5). Recent evidence has also suggested that off-pump CABG (OPCAB) has higher rates of reintervention than conventional on-pump CABG, with potentially inferior anastomoses (6). The minimally invasive approach combined with $\mathrm{OPCAB}$ is more technically challenging than OPCAB alone, so LIMALAD patency rates need to be carefully followed. We will define the role of HCR in myocardial revascularization, discuss patient selection, present results with MICSCABG and describe steps to build an HCR program with success. Finally, we will speculate on future directions and growth of the field.

\section{Methods}

\section{Patients}

Selecting appropriate patients for HCR is an important step, as not every patient with CAD is an appropriate candidate. In a multicenter observational study of HCR, $12.2 \%$ of patients who underwent diagnostic angiography were deemed appropriate for a hybrid approach (7). Table 1 demonstrates criteria to determine acceptable candidates for HCR. Several of these characteristics are angiographic in nature. For example, non-LAD lesions should be low- to intermediate-risk by SYNTAX score to improve success of PCI, minimize need for reintervention, and ideally achieve an equipoise with surgical grafting of these same vessels. Other important criteria to consider are patient-specific factors, like previous chest surgery or significant obesity, which can make minimally invasive bypass more difficulty. This approach should not be used for emergency operations, though patients presenting with an acute coronary syndrome may be good candidates for culprit vessel PCI followed by minimally invasive bypass.

\section{Interventions}

In HCR, PCI is performed according to standard institutional protocols. The surgical portion of the procedure, however, is much different from conventional CABG. There are several approaches to minimally invasive bypass, using minimally invasive direct coronary artery bypass (MIDCAB) and using robotics. In MIDCAB, a small anterior thoracotomy is made in the fourth or fifth intercostal space (8). An alternative is to perform a partial inferior sternotomy. After entering the chest and deflating the left lung, the LIMA is harvested under direct visualization with the assistance of specialized retractor which both spreads the ribs and elevates the cranial ribs to improve visualization. Once freed from the chest wall, the LIMA-LAD anastomosis is then created though the same incision with the same technique as open surgery. The stabilizer for creating an off-pump anastomosis is introduced though a separate stab incision inferiorly, which is later converted into a chest tube site.

An alternative to open harvesting of the LIMA is to endoscopically harvest it using robotic instrumentation. The da Vinci surgical platform (Intuitive Surgical, Sunnyvale, CA, USA), is used for this. A camera port and two instrument ports are introduced into the left chest in approximately the $2^{\text {nd }}, 4^{\text {th }}$, and $6^{\text {th }}$ intercostal spaces in 

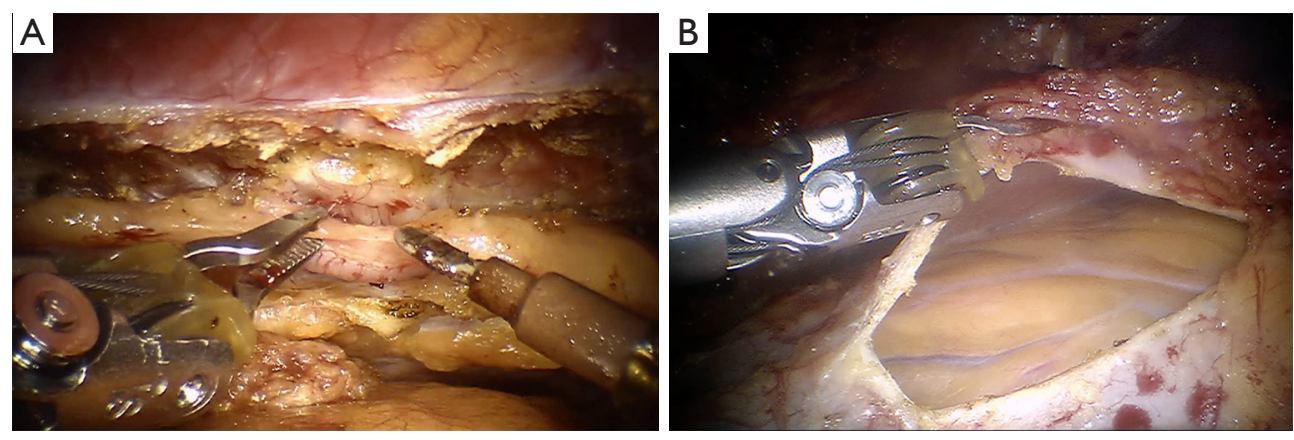

Figure 1 Components of robotic CABG operations. (A) Harvest of the LIMA; (B) identifying the LAD. CABG, coronary artery bypass grafting; LIMA, left internal mammary artery; LAD, left anterior descending artery.

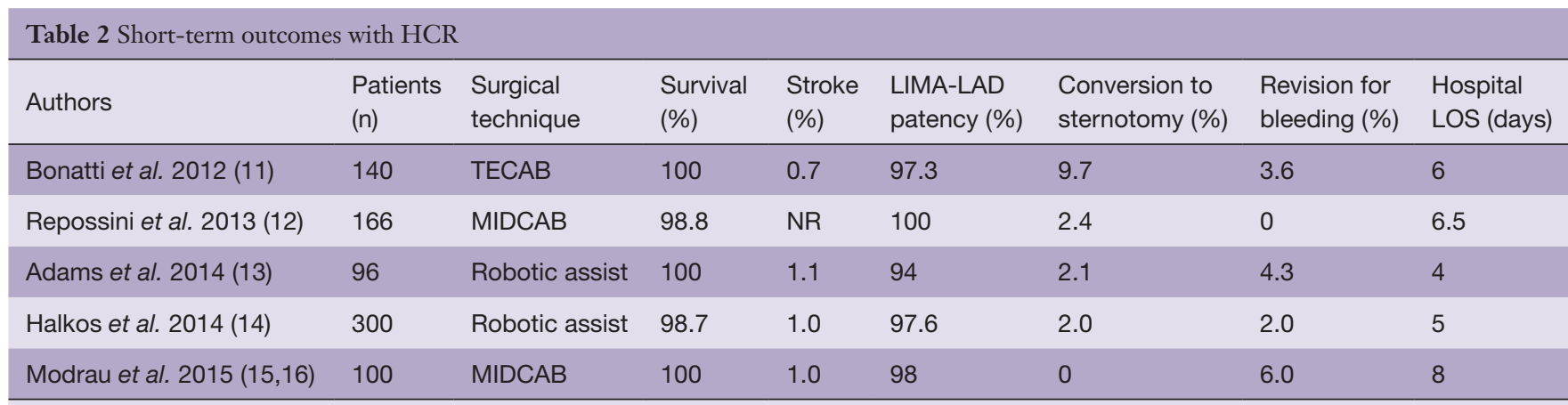

HCR, hybrid coronary revascularization; LOS, length of stay; NR, not recorded; LIMA, left internal mammary artery; LAD, left anterior descending artery; TECAB, total endoscopic coronary artery bypass; MIDCAB, minimally invasive direct coronary artery bypass.

the anterior axillary line. The LIMA is then harvested according to surgeon preference, either in a skeletonized or pedicled fashion. Next, a thoracotomy is made directly over the LAD, through which the anastomosis is carried out similar to the MIDCAB procedure (robotic assisted) (9). An alternative is total endoscopic coronary artery bypass (TECAB). In TECAB, the LIMA is harvested just as with a robotic assisted approach, but rather than make a thoracotomy and complete the anastomosis in an open fashion, a robotic stabilizer is introduced through a fourth port, and the anastomosis is then carried out using the robotic instruments (10). Figure 1 demonstrates robotic LIMA harvest and exposure of the heart for planned bypass.

\section{Results}

While HCR has been performed for 2 decades, it has steadily gained popularity over the last 7-10 years, due in part to improved PCI technologies, but perhaps mostly by the availability of a robotic surgical platform. For this reason, much of the literature to date focuses on shortterm results, though there are some reports out to 10 years. There have been two randomized trials completed, and a large multicenter trial is currently underway in the US.

\section{Short-term outcomes}

Multiple centers have reported on their outcomes with HCR in the past 5 years. These include the three main surgical techniques: MIDCAB, robotic assist, and TECAB. Table 2 shows short term outcomes of several large recent series.

While the surgical techniques employed were varied, these groups all showed relatively similar results. Patient survival was $99 \%$ with stroke at $1 \%$ or less. LIMA-LAD patency was $95 \%$ and higher across groups, with conversion to sternotomy typically $2 \%$ or less. Revision for bleeding was less than $5 \%$, and hospital length of stay was roughly 5 days.

Recovery after HCR does not approach that of PCI, where elective interventions are often performed with 
either one night in the hospital or on an outpatient basis. However, compared to conventional CABG, short-term results may be superior. Table 2 demonstrates excellent outcomes with relatively short length of stay, but are not compared to institutional conventional CABG data. HCR was compared to multi-arterial grafting in a retrospective review, where less blood transfusion and a shorter length of stay were noted; 30-day MACCE were similar (17). Other studies have shown faster recovery after surgery (18), with quicker return to work (19) and improved quality of life scores at 3 months (20).

\section{Mid-term outcomes}

Several groups have published mid-term outcomes at 5 years, and have shown rates of major adverse cardiovascular events (MACCE): 20\% (11), 17\% (12), and 21\% (13). With no comparison groups, it is somewhat difficult to compare these to patients undergoing PCI alone or conventional CABG. One group recently published 10-year outcomes after HCR using a MIDCAB approach. They showed $76 \% 10$-year survival, with only $10 \%$ of patients requiring repeat revascularization. Patient survival in this study was somewhat limited by a mean age of 64 and the fact that more than half of these patients had reduced ejection fractions and class II or higher heart failure at time of treatment (21). In a separate study comparing HCR to multivessel CABG with and without bilateral IMA usage, survival was similar at 5 years $(88.5 \%$ vs. $86.4 \%$ vs. $89.9 \%$, respectively, $\mathrm{P}=0.91$ ) (17). This study did not assess MACCE in the mid-term, however. Single-vessel MIDCAB was compared to PCI in a randomized trial of 220 patient and found to have much lower reintervention rates than PCI (10\% for MIDCAB, $32 \%$ for PCI). A meta-analysis of MIDCAB (non-hybrid) to PCI also showed a significantly lower reintervention rates with surgery (1.5\% vs. 20\%). In a recently completed hybrid observational trial in the US, HCR patients were found to have similar MACCE at 18 months to patients treated with PCI, with a trend toward higher MACCE in the PCI group in later follow-ups (7).

\section{Randomized data}

Two small randomized trials have been completed on HCR. The first, the POL-MIDES study, compared HCR to conventional CABG in 200 patients (22). In this study, no significant differences were seen in death, myocardial infarction, stroke, major bleeding, or repeat revascularization at 1 -year (MACCE $11 \%$ vs. 8\%). LIMALAD patency was equivalent ( $94 \% v s .93 \%$ ). Conversion to sternotomy was performed in $6.1 \%$ of patients.

A second trial, the HREVS trial, was recently presented at the Transcatheter Cardiovascular Therapeutics meeting in October 2017. This trial compared HCR using a MIDCAB approach to both conventional CABG and PCI in 155 patients (23). They showed that HCR was associated with less bleeding than CABG, and had a $10 \%$ conversion rate to sternotomy. The endpoint of residual myocardial ischemia by single-photon emission computed tomography (SPECT) was no different between any of the three groups at 1 year. Unfortunately, no longer-term follow up is present in either of these trials, where divergence of curves could occur as was seen in the previously mentioned nonhybrid MIDCAB vs. PCI trials.

\section{Discussion}

We have shown that excellent short-term outcomes with HCR are possible, irrespective of whether a MIDCAB or robotic approach is used. The LIMA-LAD, which is the most important benefit of CABG (4), can be performed reliably with patency greater than $95 \%$. Rates of blood transfusions are low, and the postoperative complications with HCR are generally similar but less frequent than conventional CABG. Ventilator times, ICU stay, and hospital stay, are all shown to be superior to conventional surgery in observational data. At mid-term follow-up, HCR seems to have better rates of revascularization than PCI but may not be identical to conventional CABG. Patient satisfaction is extremely high with HCR, and patients are back at work with no activity limitations much faster than after a sternotomy. Deep sternal wound infections are also completely avoided.

HCR consists of two procedures, and the timing of these is dependent upon several factors. In general, it is best to treat the culprit lesion first; most commonly in clinical practice, the surgical component is completed first (24). When PCI is performed first, antiplatelet agents must be continued during surgery, which can be associated with higher chest tube output. Performing surgery first provides protection of the completed LIMA-LAD anastomosis. When performed concurrently in a hybrid operation room, immediate assessment of the anastomosis can be performed, though is often logistically challenging to coordinate the two procedural teams.

A major weakness of data on HCR is the lack of robust 
randomized data comparing the approach to conventional surgery and PCI. Much more long-term data is needed before any firm conclusions can be made. The mortality benefit of the LIMA-LAD has been shown in open surgery, and there may never be a large trial performed to demonstrate that this same benefit applied to minimally invasive LIMA-LAD performed as part of HCR. A major limitation to the expansion of HCR is the lack of surgeons committed to the approach. While 15 years old, a survey of surgeons showed that only $10 \%$ were in favor of HCR $v$ s. $50 \%$ of cardiologists (25).

A major factor contributing to the lack of surgical backing of HCR is the difficulty of performing these procedures. MIDCAB is more technically challenging than conventional CABG. One group used a cumulative sum analysis to evaluate the learning curve for MIDCAB and found that 50-100 cases were required. They additionally showed that surgeons needed to maintain a significant case volume to maintain quality (26). The use of robotics to improve visualization and instrument precision may be associated with a lower learning curve of about 30 cases (27). The TECAB approach requires more time to learn, but surgeons can start with robotic assisted operations until comfortable, before attempting an endoscopic anastomosis.

Beyond the surgical learning curve, another key component of successfully performing HCR is to build a team with key stakeholders actively involved. This involves not just the surgical team, but includes referring cardiologists and interventionalists. Administration is also important, especially if robotics are used, as this is associated with significant fixed costs. We recommend using the same surgical team (i.e., anesthesiologist, first assistant, surgical technician, and circulator nurse) until the learning curve is complete. This will decrease variability and increase the speed of team learning. Access to a robot may need to be worked out ahead of time, as frustration will quickly develop if it is not routinely available. Buy in from hospital and operating room administration is key to this.

Patients are increasingly demanding minimally invasive approaches, and the trend toward PCI is showing no signs of abating. Growth of HCR may be one important way to combat this and provide patients the excellent longterm outcomes that are possible with a LIMA-LAD, while also benefiting from the steady improvements in stent technology. A multicenter randomized trial is now recruiting patient to compare HCR to PCI (28). A positive result from this trial may greatly increase the number of patients referred for this approach. While HCR is performed rarely at present, we believe that it can and should grow to about $10 \%$ of the market for coronary revascularization. HCR represents one element of offering patients state of the art coronary surgery.

\section{Acknowledgements}

None.

\section{Footnote}

Conflicts of Interest: Dr. Michael E. Halkos is a consultant for Medtronic. The other authors have no conflicts of interest to declare.

\section{References}

1. Serruys PW, Morice MC, Kappetein AP, et al. Percutaneous coronary intervention versus coronary-artery bypass grafting for severe coronary artery disease. N Engl J Med 2009;360:961-72.

2. Farkouh ME, Domanski M, Sleeper LA, et al. Strategies for multivessel revascularization in patients with diabetes. N Engl J Med 2012;367:2375-84.

3. Park SJ, Ahn JM, Kim YH, et al. Trial of everolimuseluting stents or bypass surgery for coronary disease. $\mathrm{N}$ Engl J Med 2015;372:1204-12.

4. Loop FD, Lytle BW, Cosgrove DM, et al. Influence of the internal-mammary-artery graft on 10-year survival and other cardiac events. N Engl J Med 1986;314:1-6.

5. Mehta RH, Ferguson TB, Lopes RD, et al. Saphenous vein grafts with multiple versus single distal targets in patients undergoing coronary artery bypass surgery: one-year graft failure and five-year outcomes from the Project of Ex-Vivo Vein Graft Engineering via Transfection (PREVENT) IV trial. Circulation 2011;124:280-8.

6. Shroyer AL, Hattler B, Wagner TH, et al. Five-Year Outcomes after On-Pump and Off-Pump CoronaryArtery Bypass. N Engl J Med 2017;377:623-32.

7. Puskas JD, Halkos ME, DeRose JJ, et al. Hybrid Coronary Revascularization for the Treatment of Multivessel Coronary Artery Disease: A Multicenter Observational Study. J Am Coll Cardiol 2016;68:356-65.

8. Benetti FJ, Ballester C, Sani G, et al. Video assisted coronary bypass surgery. J Card Surg 1995;10:620-5.

9. Halkos ME, Liberman HA, Devireddy C, et al. Early clinical and angiographic outcomes after robotic-assisted 
coronary artery bypass surgery. J Thorac Cardiovasc Surg 2014;147:179-85.

10. Bonatti J, Schachner T, Bonaros N, et al. Robotically assisted totally endoscopic coronary bypass surgery. Circulation 2011;124:236-44.

11. Bonatti JO, Zimrin D, Lehr EJ, et al. Hybrid coronary revascularization using robotic totally endoscopic surgery: perioperative outcomes and 5-year results. Ann Thorac Surg 2012;94:1920-6; discussion 1926.

12. Repossini A, Tespili M, Saino A, et al. Hybrid revascularization in multivessel coronary artery disease. Eur J Cardiothorac Surg 2013;44:288-93; discussion 293-4.

13. Adams C, Burns DJ, Chu MW, et al. Single-stage hybrid coronary revascularization with long-term follow-up. Eur J Cardiothorac Surg 2014;45:438-42; discussion 442-3.

14. Halkos ME, Walker PF, Vassiliades TA, et al. Clinical and angiographic results after hybrid coronary revascularization. Ann Thorac Surg 2014;97:484-90.

15. Modrau IS, Holm NR, Maeng M, et al. Oneyear clinical and angiographic results of hybrid coronary revascularization. J Thorac Cardiovasc Surg 2015;150:1181-6.

16. Modrau IS, Nielsen PH, Botker HE, et al. Feasibility and early safety of hybrid coronary revascularisation combining off-pump coronary surgery through J-hemisternotomy with percutaneous coronary intervention.

EuroIntervention 2015;10:e1-6.

17. Rosenblum JM, Harskamp RE, Hoedemaker N, et al. Hybrid coronary revascularization versus coronary artery bypass surgery with bilateral or single internal mammary artery grafts. J Thorac Cardiovasc Surg 2016;151:1081-9.

18. Harskamp RE, Walker PF, Alexander JH, et al. Clinical outcomes of hybrid coronary revascularization versus coronary artery bypass surgery in patients with diabetes mellitus. Am Heart J 2014;168:471-8.

19. Poston RS, Tran R, Collins M, et al. Comparison of economic and patient outcomes with minimally invasive

Cite this article as: Kayatta MO, Halkos ME, Puskas JD. Hybrid coronary revascularization for the treatment of multivessel coronary artery disease. Ann Cardiothorac Surg 2018;7(4):500-505. doi: 10.21037/acs.2018.06.09 versus traditional off-pump coronary artery bypass grafting techniques. Ann Surg 2008;248:638-46.

20. Bonaros N, Schachner T, Wiedemann D, et al. Quality of life improvement after robotically assisted coronary artery bypass grafting. Cardiology 2009;114:59-66.

21. Farid S, Ali JM, Stohlner V, et al. Long-Term Outcome of Patients Undergoing Minimally Invasive Direct Coronary Artery Bypass Surgery: A Single-Center Experience. Innovations (Phila) 2018;13:23-8.

22. Gasior M, Zembala MO, Tajstra M, et al. Hybrid revascularization for multivessel coronary artery disease. JACC Cardiovasc Interv 2014;7:1277-83.

23. HREVS: A Randomized Trial of PCI vs CABG vs Hybrid Revascularization in Patients With Coronary Artery Disease. Available online: https://www.tctmd. com/slide/hrevs-randomized-trial-pci-vs-cabg-vs-hybridrevascularization-patients-coronary-artery. Accessed $11 / 22 / 2017$.

24. Harskamp RE, Brennan JM, Xian Y, et al. Practice patterns and clinical outcomes after hybrid coronary revascularization in the United States: an analysis from the society of thoracic surgeons adult cardiac database. Circulation 2014;130:872-9.

25. D'Ancona G, Vassiliades TA, Boyd WD, et al. Is hybrid coronary revascularization favored by cardiologists or cardiac surgeons? Heart Surg Forum 2002;5:393-5.

26. Holzhey DM, Jacobs S, Walther T, et al. Cumulative sum failure analysis for eight surgeons performing minimally invasive direct coronary artery bypass. J Thorac Cardiovasc Surg 2007;134:663-9.

27. Oehlinger A, Bonaros N, Schachner T, et al. Robotic endoscopic left internal mammary artery harvesting: what have we learned after 100 cases? Ann Thorac Surg 2007;83:1030-4.

28. Hybrid Coronary Revascularization Trial. Available online: https://clinicaltrials.gov/show/NCT03089398. Accessed 11/21/2017. 